\title{
MONITORING ADVERSE EVENTS FOLLOWING IMMUNISATION
}

\section{Glenda Lawrence}

National Centre for Immunisation Research

and Surveillance of Vaccine Preventable Diseases

The University of Sydney and The Children's Hospital at Westmead

\section{Sue Campbell-Lloyd and Gay Rixon \\ AIDS-Infectious Diseases Branch \\ NSW Department of Health}

Surveillance of adverse events following immunisation (AEFIs) is an integral component of any immunisation program; it is as important as surveillance of both immunisation coverage and vaccine preventable diseases. ${ }^{1,2}$ In this article we describe the purpose of AEFI surveillance and the methods used to monitor AEFIs in Australia and NSW. We also summarise NSW AEFI surveillance reports received between January 2000 and November 2002.

\section{ROLE OF AEFI SURVEILLANCE}

Successful immunisation programs depend on the use of safe vaccines, and on a public perception of safety. Unlike drugs, which are usually used to treat individuals who are ill, vaccines are given mainly to healthy children; received by a very high proportion of the population; usually given on government recommendation; and their purpose is to prevent disease rather than to treat illness. ${ }^{3}$ As the incidence of vaccine-preventable diseases has declined due to the successful use of vaccines, public perceptions about vaccine safety and the risk of side-effects have gained prominence. While all vaccines licensed in Australia must meet strict standards of manufacture and safety evaluation, like all therapeutic agents, vaccines cannot be guaranteed to be 100 per cent safe.

The term 'AEFI' is recommended by the World Health Organization (WHO) to describe any immunisation-related adverse event. ${ }^{4}$ AEFI describes any adverse event related to a vaccine or to its handling or administration. The term also encompasses the concept that an adverse event may be associated coincidentally with the timing of immunisation without necessarily being causally linked to either the vaccine or the immunisation process.

The primary purpose of AEFI surveillance is to detect rare, late onset, unexpected and population-specific adverse events that cannot be detected in the pre-licensure vaccine trials due to the numbers enrolled in the trials, the timeframe of follow-up, or in different populations or age groups. Routine ongoing monitoring of AEFIs after vaccine licensure also helps to identify specific problems related to vaccine manufacture, storage or administration (for example, batch contamination, freezing of vaccine and incorrect diluent). It allows detection of changes in AEFIs over time (for example, following the change from whole-cell to acellular pertussis vaccines). The maintenance and reporting of data specific to each country helps to maintain local public confidence in immunisation programs. ${ }^{3,4}$

Recent examples of AEFIs detected by post-licensure surveillance include excessive limb swelling after a fourth or fifth dose of acellular pertussis-containing vaccines, 5,6 and intussusception in infants who received the rotavirus vaccine in the USA. ${ }^{7}$ The rotavirus vaccine was withdrawn from the market six months after licensure. The excessive limb swelling after acellular pertussis vaccines has been studied extensively; it resolves without sequelae and the benefit of pertussis vaccination far outweighs the risk of this adverse event. ${ }^{5,6}$

\section{AEFI surveillance methods}

AEFI surveillance systems, worldwide and in Australia, are usually passive systems that rely on health service providers and members of the public notifying suspected AEFIs. ${ }^{3,4,8,9}$ Data are monitored to identify 'signals' above background 'noise', and to identify events clustered by vaccine, time or place. If a signal or cluster is detected, specific epidemiological studies can be instituted to investigate these further. Under-reporting, particularly of less serious AEFIs, is a limitation. Passive surveillance is complemented by specialist clinics, ${ }^{5}$ which function as sentinel surveillance sites for more serious AEFIs, enhanced surveillance during ad-hoc immunisation campaigns such as the 1998 measles catch-up campaign, ${ }^{10}$ and active surveillance methods such as the Vaccine Safety Datalink project in the USA. ${ }^{11}$

Passive AEFI surveillance mechanisms differ for each Australian state and territory. ${ }^{9}$ However, all rely on doctors, other health professionals and parents to report suspected AEFIs to a relevant authority and encourage reporting of specific conditions listed in the Australian Immunisation Handbook. ${ }^{9}$ In NSW, doctors are required under the NSW Public Health Act (1991) to notify their local public health unit (PHU) of suspected AEFIs. The purpose of mandatory notification in NSW is to reduce under-reporting of suspected AEFIs by medical practitioners, and to allow individual case investigation by PHU staff. Other health service professionals and parents are also strongly encouraged to notify their local PHU of suspected AEFIs.

All AEFI reports from each jurisdiction are forwarded to the Adverse Drug Reactions Unit at the Therapeutic Goods Administration in Canberra for collation, review and analysis. Reports are assessed to determine the likelihood of a reaction being causally associated with the vaccine(s) administered. The criteria used to define the different levels of causality (certain, probable, possible, unclear, unknown) allow comparison with international AEFI data. All Australian data are also reviewed by the Adverse Drug Reactions Advisory Committee (ADRAC), which has 


\section{FIGURE 1}

AGE AND GENDER DISTRIBUTION OF PEOPLE REPORTED WITH SUSPECTED ADVERSE EVENTS FOLLOWING IMMUNISATION, NSW, 1 JANUARY 2000-8 NOVEMBER 2002

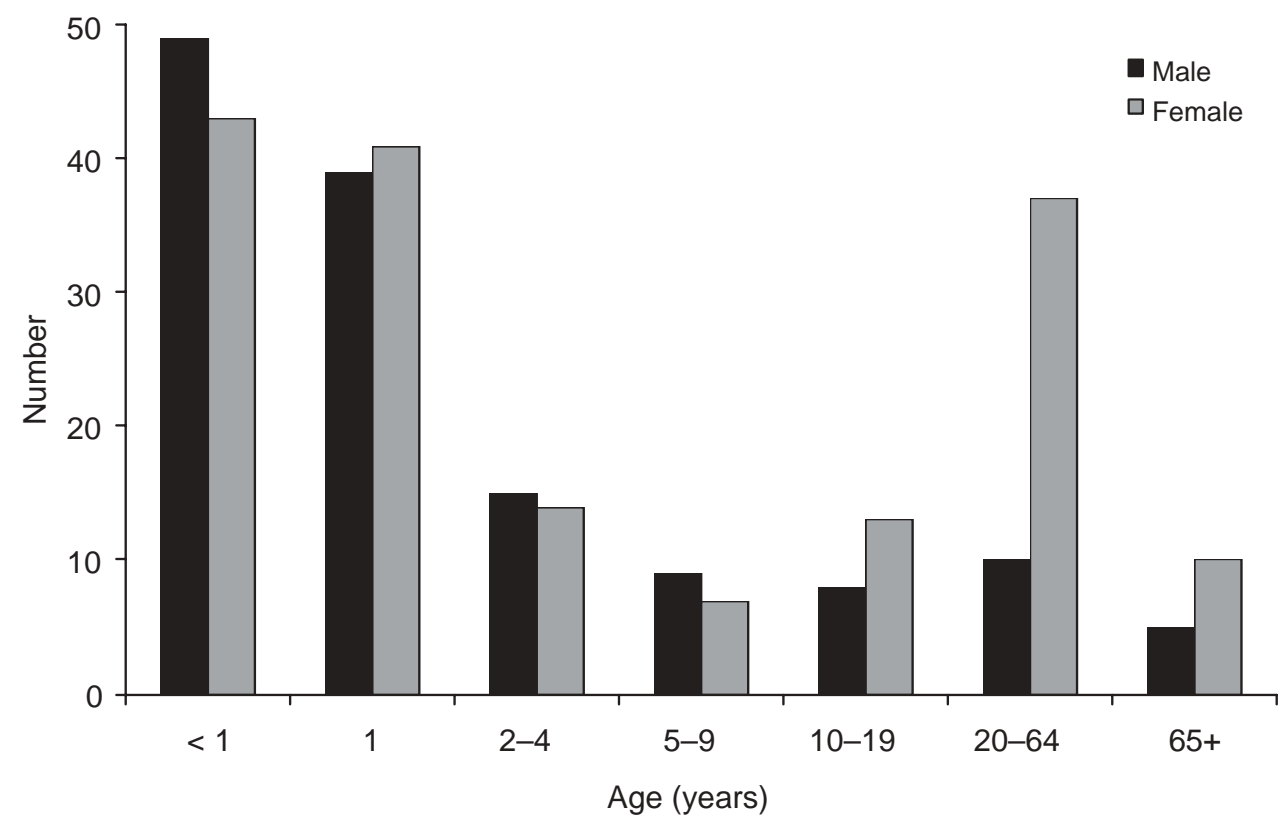

Source: Notifiable Diseases Database (NDD), NSW Department of Health

\section{FIGURE 2}

DISTRIBUTION OF SUSPECTED ADVERSE EVENTS FOLLOWING IMMUNISATION, BY MOST SERIOUS REACTION REPORTED, NSW, 1 JANUARY 2000-8 NOVEMBER 2002

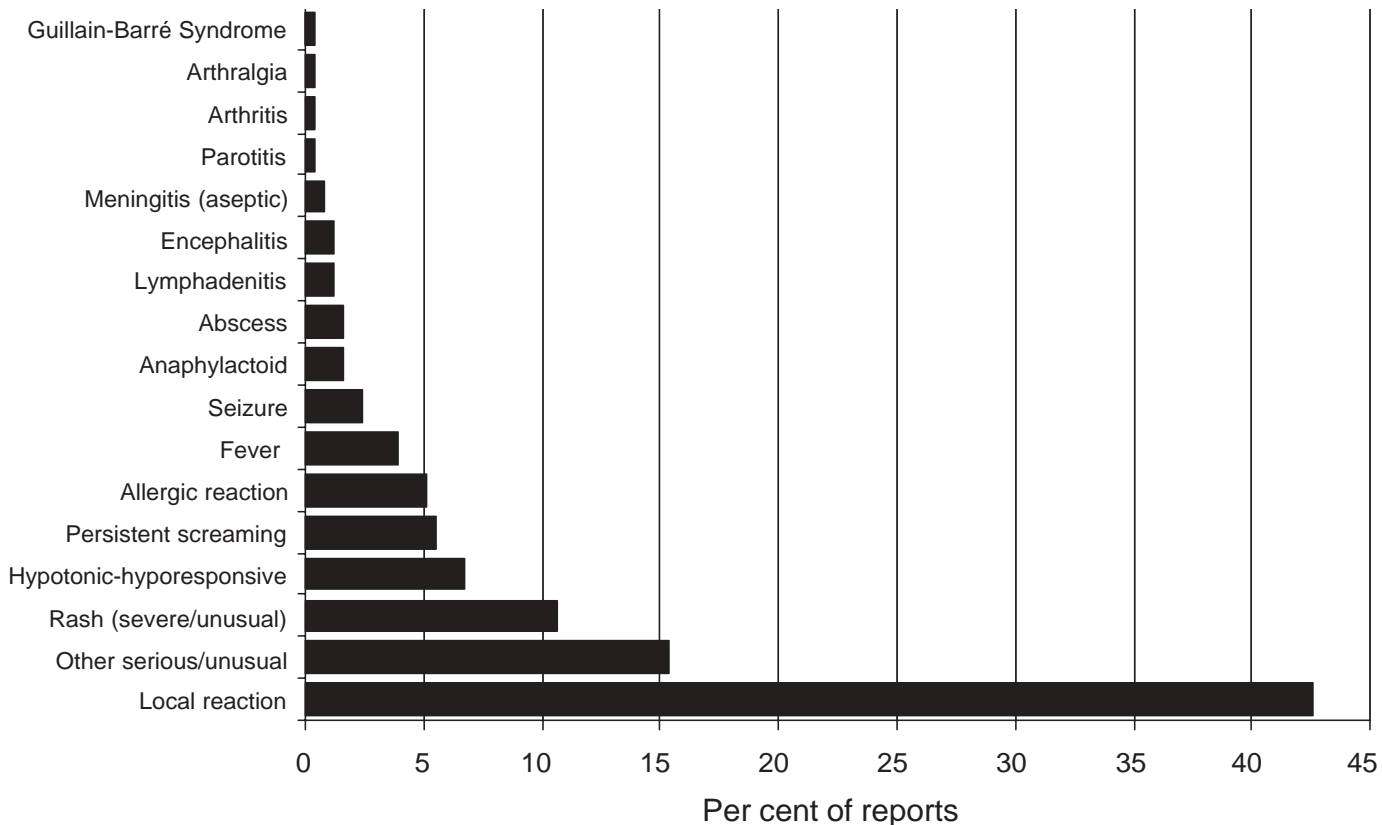

Note: The category of 'other severe-unusual' reactions included a wide range of reactions, such as bradycardia with apnoea, chest tightness, vomiting, lethargy, dizziness, and muscle spasms.

Source: Notifiable Diseases Database (NDD), NSW Department of Health 
FIGURE 3

DISTRIBUTION OF VACCINE TYPES INCLUDED IN 301 REPORTS OF SUSPECTED ADVERSE EVENTS FOLLOWING IMMUNISATION, BY NUMBER OF VACCINES ADMINISTERED AT THE SAME VACCINATION EPISODE, NSW, 1 JANUARY 2000-8 NOVEMBER 2002

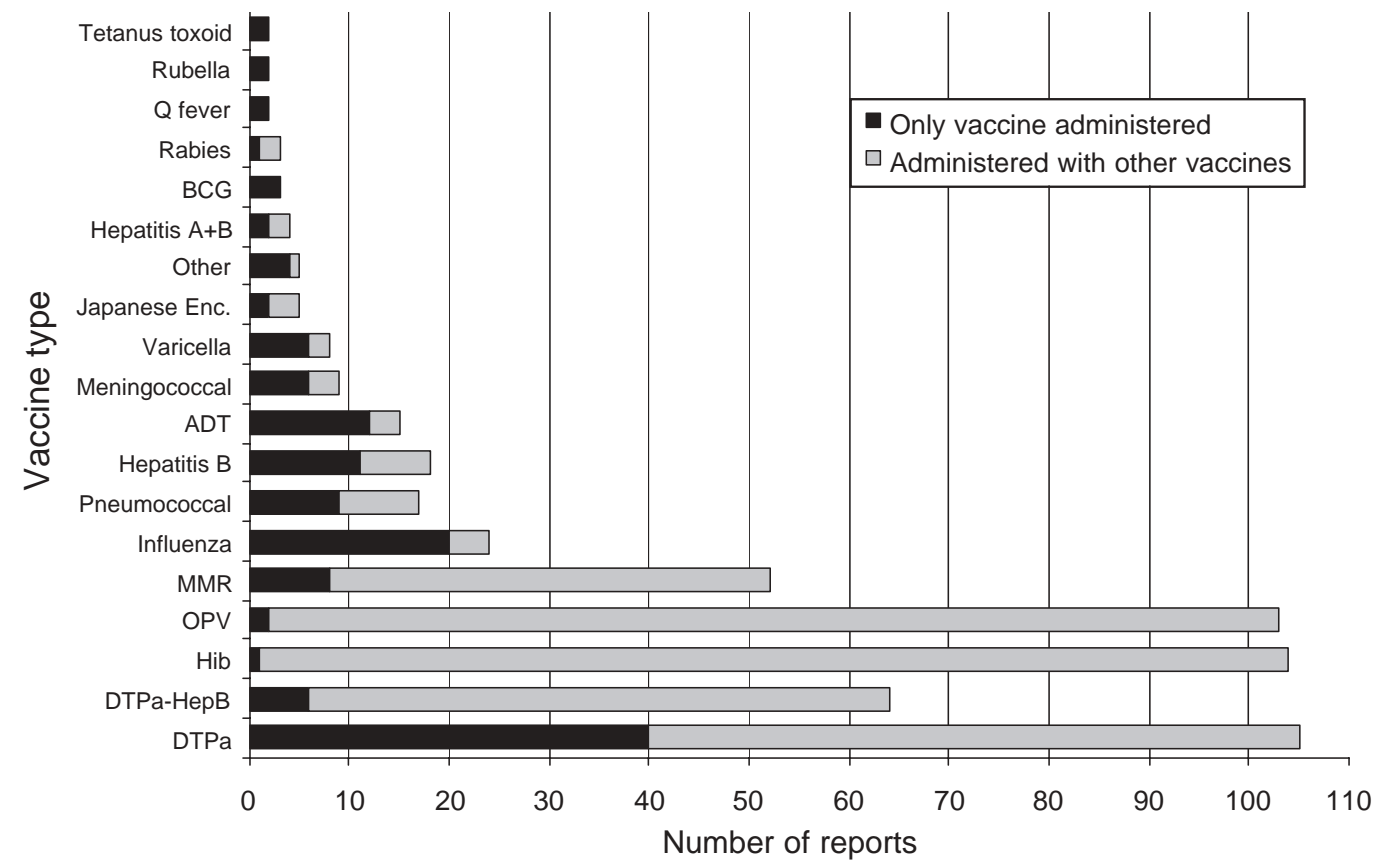

Note: $\quad$ BCG $=$ Bacille Calmette-Guèrin; Japanese Enc = Japanese Encephalitis; $A D T=$ adult diphtheria-tetanus; MMR = measles-mumps-rubella; OPV = oral poliomyelitis vaccine; Hib = Haemophilus influenzae type b; DTPa-HepB = diphtheriatetanus-acellular pertussis combined with hepatitis B; DTPa = diphtheria-tetanus-acellular pertussis. 'Other' category included one report each of inactivated poliomyelitis vaccine (IPV), combined diphtheria-tetanus (CDT) vaccine, yellow fever vaccine, hepatitis B immunoglobulin and purified protein derivative (PPD).

Source: Notifiable Diseases Database (NDD), NSW Department of Health

overall responsibility for AEFI surveillance in Australia. Summary data are reported to the WHO.

\section{NSW AEFI REPORTS SINCE 2000}

AEFIs notified to NSW PHU staff are entered into the NSW Notifiable Diseases Database (NDD). Three hundred and one AEFI reports were received between 1 January 2000 and 8 November 2002. The majority were for children under two years of age (Figure 1). The gender ratio differed by age group, with slightly more males in the younger age groups and more females in the adolescent and adult age groups. The most frequent AEFIs reported were local reactions, other serious or unusual reactions, rashes and hypotonic-hyporesponsive episodes (HHEs) (Figure 2).

The vaccines most commonly included in AEFI reports were those recommended in the early childhood vaccination schedule, due between two and 18 months of age (Figure 3). These were diphtheria-tetanus-acellular pertussis (DTPa) vaccine alone or combined with hepatitis $\mathrm{B}$ vaccine (DTPa-HepB), oral poliomyelitis vaccine (OPV) and Haemophilus influenzae type B (Hib) vaccine. The majority of reports involved a DTPa vaccine plus Hib and OPV, reflecting the fact that these vaccines are usually given at the same time to young children. When more than one antigen is given at the same vaccination episode, it is not possible to identify which antigen might have caused the reported AEFI. Older age groups were more likely to report a suspected AEFI following receipt of a single antigen vaccine. In all age groups, the pertussis, diphtheria and tetanus-containing vaccines were most commonly associated with local reactions.

\section{CONCLUSION}

Surveillance of suspected AEFIs is an integral component of the national and NSW immunisation programs. As with all passive surveillance systems, under-reporting is likely to occur, although this is less of an issue for the most serious AEFIs. Over a period of almost three years, there were 301 reports of AEFIs to the NSW Department of Health, the majority of which were either local reactions 
or less serious systemic reactions. During the same time period, approximately one million NSW children and adults received several million vaccinations. Vaccination coverage among children under two years of age has risen to over 90 per cent, ${ }^{12}$ while the incidence of diseases such as measles and Hib meningitis has declined markedly in the past decade. ${ }^{2}$ Continued effort on the part of immunisation service providers, public health practitioners and other health care workers is necessary to sustain and improve AEFI surveillance in Australia.

\section{ACKNOWLEDGEMENTS}

We thank everyone who has reported suspected AEFIs to the NSW Department of Health, and all PHU staff involved in AEFI surveillance.

\section{REFERENCES}

1. Hull BP, McIntyre PB. Mapping immunisation coverage and conscientious objectors to immunisation in NSW. NSW Public Health Bull 2002;13(12).

2. McIntyre P, Gidding H, Gilmour R, Lawrence G, Hull B, Horby P, et al. Vaccine Preventable Diseases and Vaccination Coverage in Australia, 1999-2000. Commun Dis Intell 2002; Suppl: www.health.gov.au/pubhlth/cdi/pubs/pdf/vpd9900.pdf Accessed 2 December 2002.

3. Ellenberg SS, Chen RT. The complicated task of monitoring vaccine safety. Public Health Reports. Jan-Feb 1997;112:10-19: www.cdc.gov/nip/vacsafe/ research/phr.htm Accessed 2 December 2002.

4. Mansoor O, Shin S, Maher C, Immunization Focus of WPRO. Immunisation safety surveillance: guidelines on reporting and investigating adverse events following immunization. Manila: World Health Organization Regional Office for the Western Pacific, 1999; http://whqlibdoc.who.int/wpro/199499/WPRO_EPI_99.01.pdf Accessed 2 December 2002.

5. Wood N. Immunisation adverse events clinics. N S W Public Health Bull 2002;13(12).

6. Rennels MB, Deloria MA, Pichichero ME, Losonsky GA, Englund JA, Meade BD, et al. Extensive swelling after booster doses of acellular pertussis-tetanus-diphtheria vaccines. Pediatrics 2000;105:e12.

7. Murphy TV, Gargiullo PM, Massoudi MS, Nelson DB, Jumaan AO, Okoro CA, et al. Rotavirus Intussusception Investigation Team. Intussusception among infants given an oral rotavirus vaccine. $N$ Engl J Med 2001;344:564-72 [erratum appears in N Engl J Med 2001;344:1564].

8. Lankinen KS, editor. Scientific and Technical Evaluation of Vaccination Programmes in the European Union. EUVAX Project Report. Helsinki: PSR Consulting Ltd, 2001.

9. National Health and Medical Research Council. The Australian Immunisation Handbook, 7th edition. Canberra: AGPS; 2000.

10. Burgess MA, Heath TC, McIntyre PB. The Measles Control Campaign and immunisation adverse events. Commun Dis Intell 1998;2:136-8.

11. Chen RT, Glasser JW, Phodes PH, Davis RL, Barlow WE, Thompson RS, et al. Vaccine safety datalink project: A new tool for improving vaccine safety monitoring in the United States. Pediatrics 1997;99:765-73.

12. Hull B, Lawrence G, MacIntyre CR, McIntyre P. Immunisation coverage: Australia 2001. Canberra: Commonwealth Department of Health and Ageing, 2002; www.health.gov.au/pubhlth/immunise/report.pdf Accessed 2 December 2002. 